\title{
Paper
}

\section{Fractional-step Tow-Thomas biquad filters}

\author{
Todd J. Freeborn ${ }^{1 a)}$, Brent Maundy ${ }^{1}$, and Ahmed Elwakil ${ }^{2 b)}$ \\ ${ }^{1}$ Department of Electrical and Computer Engineering, University of Calgary \\ 2500 University Drive N.W., Calgary, Alberta, Canada \\ ${ }^{2}$ Department of Electrical and Computer Engineering, University of Sharjah \\ P.O. Box 27272, United Arab Emirates \\ a) tjfreebo@ucalgary.ca \\ b)elwakil@ieee.org
}

Received November 12, 2011; Revised February 9, 2012; Published July 1, 2012

\begin{abstract}
In this paper we propose the use of fractional capacitors in the Tow-Thomas biquad to realize both fractional lowpass and asymmetric bandpass filters of order $0<\alpha_{1}+\alpha_{2} \leq 2$, where $\alpha_{1}$ and $\alpha_{2}$ are the orders of the fractional capacitors and $0<\alpha_{1,2} \leq 1$. We show how these filters can be designed using an integer-order transfer function approximation of the fractional capacitors. MATLAB and PSPICE simulations of first order fractional-step low and bandpass filters of order 1.1, 1.5, and 1.9 are given as examples. Experimental results of fractional low pass filters of order 1.5 implemented with silicon-fabricated fractional capacitors verify the operation of the fractional Tow-Thomas biquad.
\end{abstract}

Key Words: fractional calculus, fractional filters, fractional-order circuits, Tow-Thomas biquad

\section{Introduction}

The use of fractional calculus, the branch of mathematics concerning differentiations and integrations to non-integer order, has been steadily migrating from the theoretical realms of mathematicians into many applied branches of engineering [1]. With a fractional derivative of order $\alpha$ and initial conditions $a$ is given by the Grünwald-Letnikov approximation [2] as

$$
{ }_{a} D^{\alpha} f(x)=\lim _{h \rightarrow 0} \frac{1}{h^{\alpha}} \sum_{m=0}^{\left[\frac{x-a}{h}\right]}(-1)^{m} \frac{\Gamma(\alpha+1)}{m ! \Gamma(\alpha-m+1)} f(x-m h)
$$

where $\Gamma(\cdot)$ is the gamma function. While there are no physical analogies to these derivatives, like slope or area under the curve, they are still applicable to physical systems with varying applications including materials theory [3,4], control theory $[5,6]$, electromagnetics $[7]$, robotics $[8,9]$ and many more. The import of these concepts into circuit theory is relatively new [10] with much recent progress regarding filter theory [11,12], analysis [13] and implementation [14-16]. This emerging field has introduced fractional calculus into analog filter design to achieve continuous time filtering circuits with fractional step stopband attenuations. These circuits have been designed using the fractional 


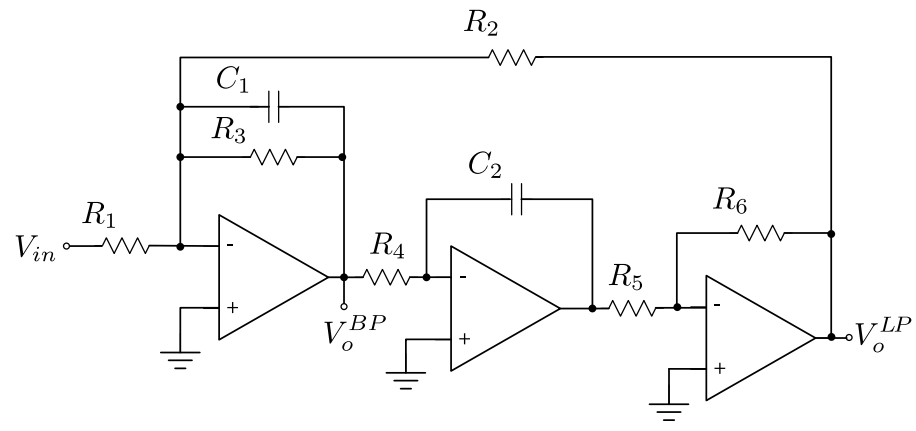

Fig. 1. Tow-Thomas biquad topology.

Laplacian operator, achieved when applying the Laplace transform to the general fractional derivative of (1) with zero initial conditions yielding

$$
\mathscr{L}\left\{{ }_{0} D^{\alpha} f(t)\right\}=s^{\alpha} F(s)
$$

The fractional Laplacian operator is especially useful in the design of filters with fractional characteristics, as the design of transfer functions can be done algebraically rather than through solving the difficult time domain representations of the fractional derivatives.

In this paper we propose replacing the capacitors of the well known Tow-Thomas biquad (see Fig. 1) with fractional capacitors in order to realize lowpass and asymmetric bandpass fractional-step filters. MATLAB and PSPICE simulated lowpass and bandpass filters having fractional steps of 1.1, 1.5, and 1.9 are presented. An experimental 1.5 order fractional low pass filter, implemented using an actual silicon-based fractional capacitor, verifies the operation of the fractional Tow-Thomas biquad.

\section{Tow-Thomas biquad}

The Tow-Thomas biquad [17-19] of Fig. 1 is an active-RC topology used to realize both lowpass and bandpass biquadratic filtering functions. This topology has been widely used because it is simple, versatile, and requires few components [17]. Analysis of this biquad yields the lowpass and bandpass functions

$$
\begin{aligned}
\frac{V_{o}^{L P}(s)}{V_{i n}(s)} & =-\frac{\frac{R_{6}}{R_{1} R_{4} R_{5} C_{1} C_{2}}}{s^{2}+\frac{s}{R_{3} C_{1}}+\frac{R_{6}}{R_{2} R_{4} R_{5} C_{1} C_{2}}} \\
\frac{V_{o}^{B P}(s)}{V_{i n}(s)} & =-\frac{s \frac{1}{R_{1} C_{1}}}{s^{2}+\frac{s}{R_{3} C_{1}}+\frac{R_{6}}{R_{2} R_{4} R_{5} C_{1} C_{2}}}
\end{aligned}
$$

While the traditional Tow-Thomas Biquad uses standard capacitors we can further generalize the filter responses realized with this circuit by introducing fractional capacitors for both $C_{1}$ and $C_{2}$. This approach of replacing traditional capacitors with fractional capacitors has previously been investigated for both the Sallen-Key filter and the Kerwin-Huelsman-Newcomb biquad [12]. However, the work in [12] examined the special case when fractional capacitors of the same order were used. Here, we replace both $C_{1}$ and $C_{2}$ in the Tow-Thomas Biquad with fractional capacitors of impedance $Z_{C_{n}}=\frac{1}{s^{\alpha_{n}} C_{n}}$, where $n \in[1,2], 0<\alpha_{n} \leq 1$, and $\alpha_{1} \neq \alpha_{2}$ for all cases. This modification results in the following transfer functions at the lowpass and bandpass outputs

$$
\begin{aligned}
& T_{F L P}(s)=\frac{V_{o}^{L P}(s)}{V_{i n}(s)}=-\frac{a}{s^{\alpha_{1}+\alpha_{2}}+b s^{\alpha_{2}}+c} \\
& T_{F B P}(s)=\frac{V_{o}^{B P}(s)}{V_{i n}(s)}=-\frac{a s^{\alpha_{2}}}{s^{\alpha_{1}+\alpha_{2}}+b s^{\alpha_{2}}+c}
\end{aligned}
$$

The transfer functions (5) and (6) realize fractional lowpass and fractional bandpass filter responses, respectively, of order $\left(\alpha_{1}+\alpha_{2}\right)$ with the coefficients $a, b$, and $c$ given in Table I. This topology provides both fractional lowpass and bandpass filter responses from the same circuit, not possible in previous realizations of fractional filters using Single Amplifier Biquads (SABs) [20]. 
Table I. Coefficients for fractional low and band pass filters described by (5) and (6).

\begin{tabular}{|c|c|c|}
\hline \multirow{2}{*}{ Coefficient } & \multicolumn{2}{|c|}{ Fractional Filter Response } \\
\cline { 2 - 3 } & Low Pass & Band Pass \\
\hline \hline$a$ & $\frac{R_{6}}{R_{1} R_{4} R_{5} C_{1} C_{2}}$ & $\frac{1}{R_{1} C_{1}}$ \\
\hline$b$ & \multicolumn{2}{|c|}{$\frac{1}{R_{3} C_{1}}$} \\
\hline$c$ & $\frac{R_{6}}{R_{2} R_{4} R_{5} C_{1} C_{2}}$ \\
\hline
\end{tabular}

Table II. Coefficients for fractional low and bandpass magnitude responses described by (7) and (10).

\begin{tabular}{|c|c|}
\hline Coefficient & Value \\
\hline \hline$x_{0}$ & $2 c \cos \left(\frac{\left(\alpha_{1}+\alpha_{2}\right) \pi}{2}\right)$ \\
\hline$x_{1}$ & $2 b c \cos \left(\frac{\alpha_{2} \pi}{2}\right)$ \\
\hline$x_{2}$ & $2 b \cos \left(\frac{\alpha_{1} \pi}{2}\right)$ \\
\hline$x_{3}$ & $b^{2}$ \\
\hline$x_{4}$ & $c^{2}$ \\
\hline$x_{5}$ & 1 \\
\hline
\end{tabular}

The two additional design parameters, $\alpha_{1}$ and $\alpha_{2}$, increase the range of filter responses that can be realized using the Tow-Thomas biquad. Whereas the traditional Tow-Thomas can only realize $2^{\text {nd }}$ order lowpass and bandpass transfer functions, the fractional Tow-Thomas can realize any order $\alpha_{1}+\alpha_{2}$ where $0<\alpha_{1}+\alpha_{2} \leq 2$.

\subsection{Fractional Lowpass Filter (FLPF)}

The FLPF described by (5) can be used to realize a low pass filter response with DC gain of $a / c$, high frequency gain of zero, and fractional attenuations of $-20\left(\alpha_{1}+\alpha_{2}\right) \mathrm{dB} /$ decade in the stopband. From the transfer function (5) the magnitude and phase responses of this circuit are given by

$$
\begin{aligned}
\left|T_{F L P F}(j \omega)\right| & =\frac{a}{\sqrt{x_{0} \omega^{\alpha_{1}+\alpha_{2}}+x_{1} \omega^{\alpha_{2}}+x_{2} \omega^{\alpha_{1}+2 \alpha_{2}}+x_{3} \omega^{2 \alpha_{2}}+x_{4}+x_{5} \omega^{2\left(\alpha_{1}+\alpha_{2}\right)}}} \\
\measuredangle T_{F L P F}(j \omega) & =\tan ^{-1}\left(-\frac{\omega^{\alpha_{1}+\alpha 2} \sin \left(\frac{\left(\alpha_{1}+\alpha_{2}\right) \pi}{2}\right)+b \omega^{\alpha_{2}} \sin \left(\frac{\alpha_{2} \pi}{2}\right)}{\omega^{\alpha_{1}+\alpha_{2}} \cos \left(\frac{\left(\alpha_{1}+\alpha_{2}\right) \pi}{2}\right)+b \omega^{\alpha_{2}} \cos \left(\frac{\alpha_{2} \pi}{2}\right)+c}\right)
\end{aligned}
$$

with the values of $x_{n}$, where $n \in[0,5]$, given in Table II. The MATLAB simulated magnitude and phase responses of (5) with fractional orders of $\left(\alpha_{1}+\alpha_{2}\right)=1.1,1.5$ and 1.9 when $\alpha_{1}=a=b=c=1$ compared to the first and second order Butterworth responses are illustrated in Figs. 2(a) and 2(b), respectively. From the magnitude response we see that the simulated stopband attenuations perfectly match the theoretical ones with attenuations of $-22,-30$, and $-38 \mathrm{~dB} /$ decade for the $1.1,1.5$, and 1.9 order FLPFs, respectively. We also note the fractional step of the phase responses between the first and second order Butterworth responses with high frequency phases of $-99^{\circ},-135^{\circ}$ and $-171^{\circ}$, matching those of $-90\left(\alpha_{1}+\alpha_{2}\right)^{\circ}$ predicted by (8) when $\omega=\infty$. The FLPF described by (5) not only offers the ability to design filters with a fractional step through the stopband but also to shape the passband response based on the selection of $\alpha_{1}$ and $\alpha_{2}$. To illustrate, the magnitude and phase response of FLPFs of order $\left(\alpha_{1}+\alpha_{2}\right)=1.5$ simulated in MATLAB while varying $\alpha_{1}$ and $\alpha_{2}$ from 1 to 0.5 and 0.5 to 1 , respectively, in steps of 0.1 are shown in Figs. 3(a) and 3(b). From Fig. 3(a) we note that as $\alpha_{1}$ decreases from 1 to 0.5 and $\alpha_{2}$ increases from 0.5 to 1 , the attenuation in the passband flattens and the roll-off increases while maintaining the same stop band atteunation. Also, from Fig. 3(b) we note that the phase response over the passband frequency range changes in the same manner as the magnitude.

From Figs. 2(a) and 3(a) it is clear that FLPFs do not share the same half power or -3dB frequency, $\omega_{-3 d B}$. The location of $\omega_{-3 d B}$ may be obtained by solving the equation 


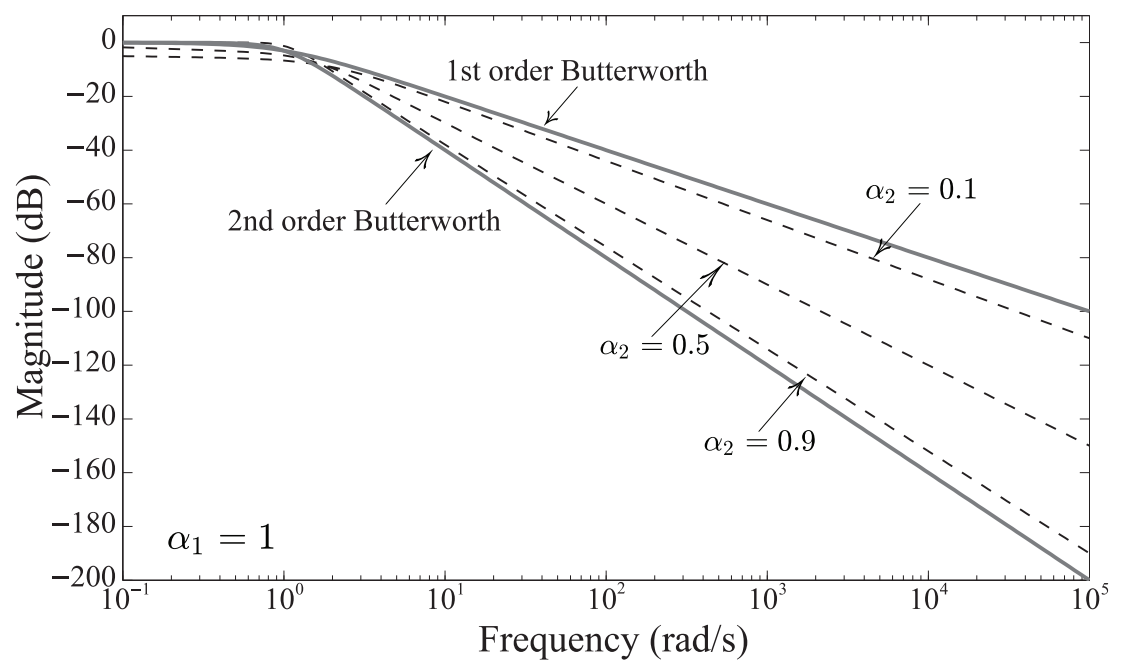

(a)

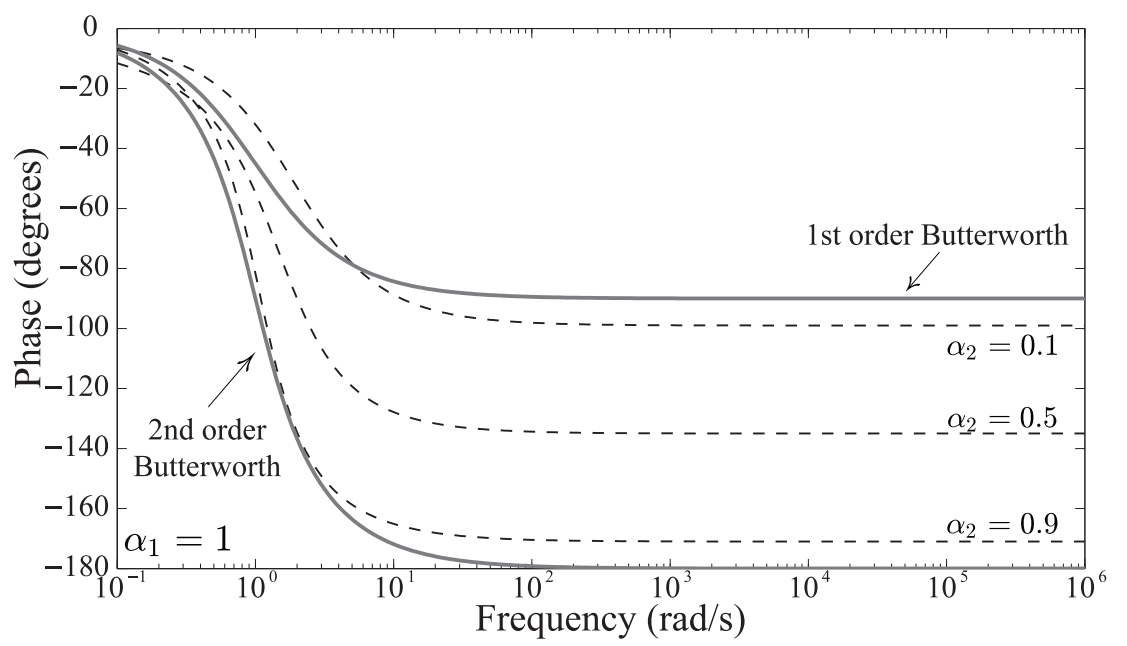

(b)

Fig. 2. (a) Magnitude and (b) phase responses of FLPFs of order $\left(\alpha_{1}+\alpha_{2}\right)=$ $1.1,1.5$ and 1.9 when $\alpha_{1}=a=b=c=1$.

$$
\begin{aligned}
0= & x^{2\left(\alpha_{1}+\alpha_{2}\right)}+2 x^{\alpha_{1}+\alpha_{2}} c \cos \left(\frac{\left(\alpha_{1}+\alpha_{2}\right) \pi}{2}\right)+2 x^{\alpha_{1}+2 \alpha_{2}} b \cos \left(\frac{\alpha_{1} \pi}{2}\right) \\
& +x^{2 \alpha_{2}} b^{2}+2 x^{\alpha_{2}} b c \cos \left(\frac{\alpha_{2} \pi}{2}\right)-c^{2}
\end{aligned}
$$

for the positive real root where $x=\omega_{-3 d B}$. The half power frequencies numerically calculated using (9) when $0<\alpha_{1,2} \leq 1$ incremented in steps of 0.05 and $a=b=c=1$ are given in Fig. 4 . We note that the half power frequencies range from $9.85 \times 10^{-11} \mathrm{rad} / \mathrm{s}$ when $\alpha_{1}=\alpha_{2}=0.05$ to $1.272 \mathrm{rad} / \mathrm{s}$ when $\alpha_{1}=\alpha_{2}=1$.

\subsection{Asymmetric Fractional Band pass Filter (FBPF)}

The FBPF described by (6) can be used to realize a fractional bandpass filter with low and high frequency gains of zero and stopband attentions of $-20 \alpha_{2}$ and $-20 \alpha_{1} \mathrm{~dB} /$ decade for frequencies lower and higher, respectively, than the center frequency. Therefore, this fractional transfer function can realize bandpass filters with asymmetric bandpass characteristics. From the transfer function (6) the magnitude and phase responses of this circuit are given by

$$
\begin{aligned}
\left|T_{F B P F}(j \omega)\right| & =\frac{a}{\sqrt{x_{0} \omega^{\alpha_{1}-\alpha_{2}}+\frac{x_{1}}{\omega^{\alpha_{2}}}+x_{2} \omega^{\alpha_{1}}+x_{3}+\frac{x_{4}}{\omega^{2 \alpha_{2}}}+x_{5} \omega^{2 \alpha_{1}}}} \\
\measuredangle T_{F B P F}(j \omega) & =\tan ^{-1}\left(-\frac{\omega^{\alpha_{1}} \sin \left(\frac{\alpha_{1} \pi}{2}\right)-\frac{c}{\omega^{\alpha_{2}}} \sin \left(\frac{\alpha_{2} \pi}{2}\right)}{\omega^{\alpha_{1}} \cos \left(\frac{\alpha_{1} \pi}{2}\right)+\frac{c}{\omega^{\alpha_{2}}} \cos \left(\frac{\alpha_{2} \pi}{2}\right)+b}\right)
\end{aligned}
$$




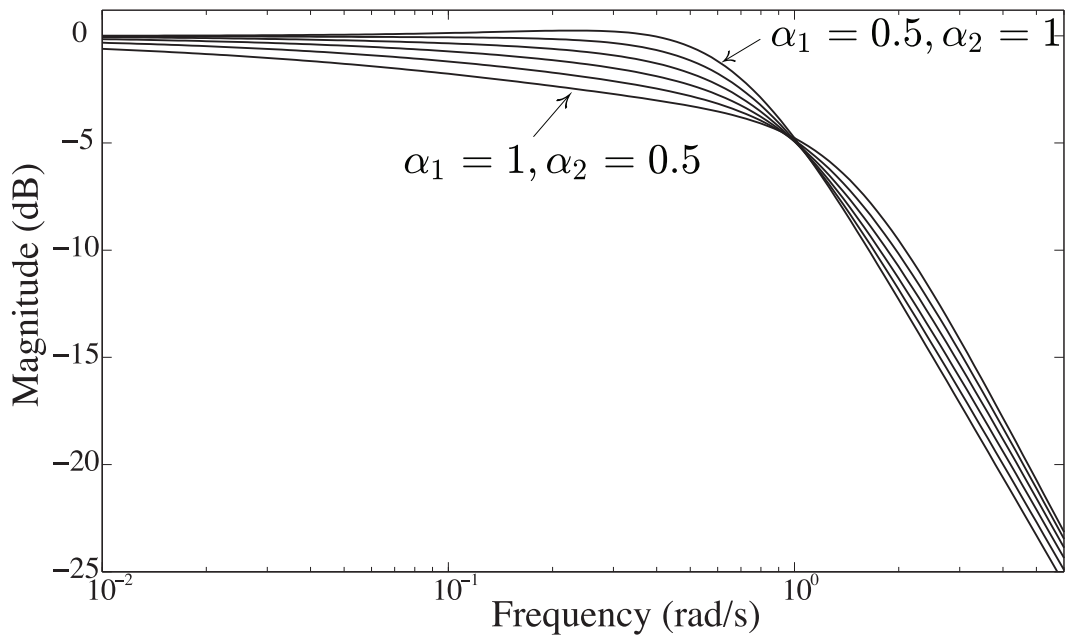

(a)

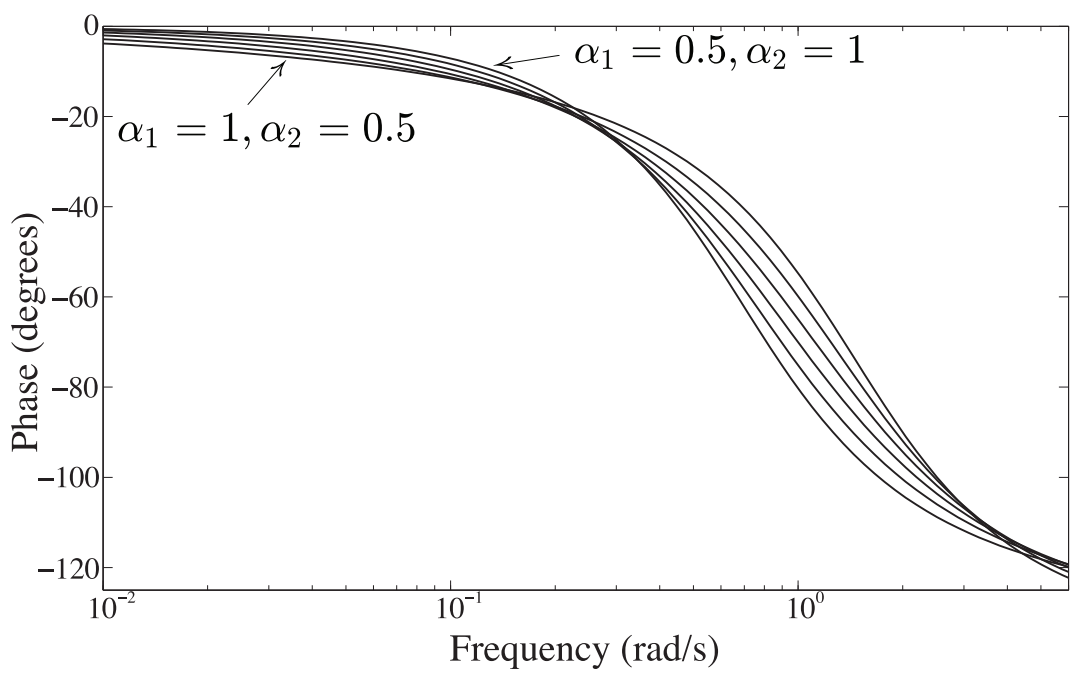

(b)

Fig. 3. (a) Magnitude and (b) phase responses of FLPFs of order $\left(\alpha_{1}+\alpha_{2}\right)=$ 1.5 varying $\alpha_{1}$ and $\alpha_{2}$ from 1 to 0.5 and 0.5 to 1 , respectively, in steps of -0.1 and 0.1 , respectively, when $a=b=c=1$.

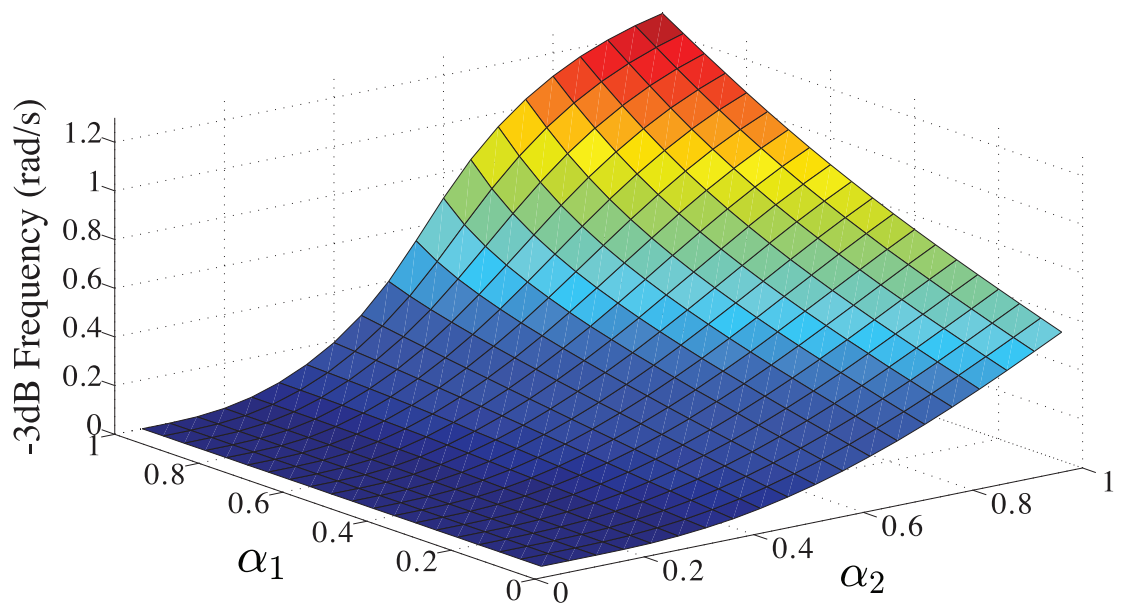

Fig. 4. Calculated half power frequencies of FLPFs using (9) when $0<\alpha_{1,2} \leq$ 1 in steps of 0.05 and $a=b=c=1$. 


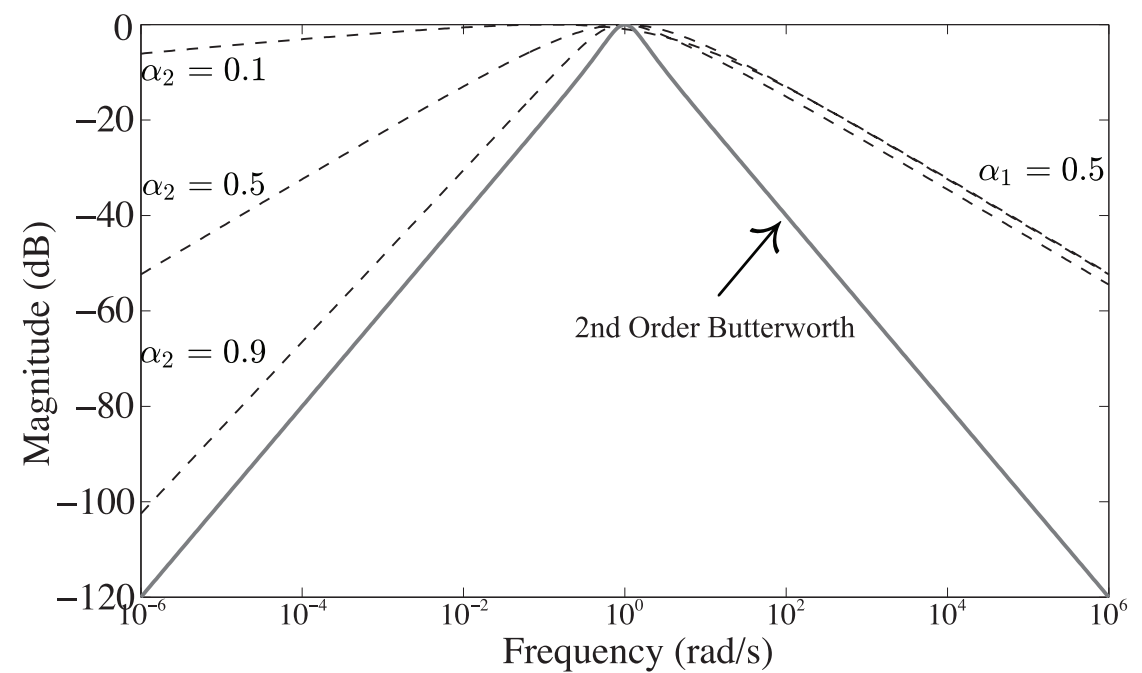

(a)

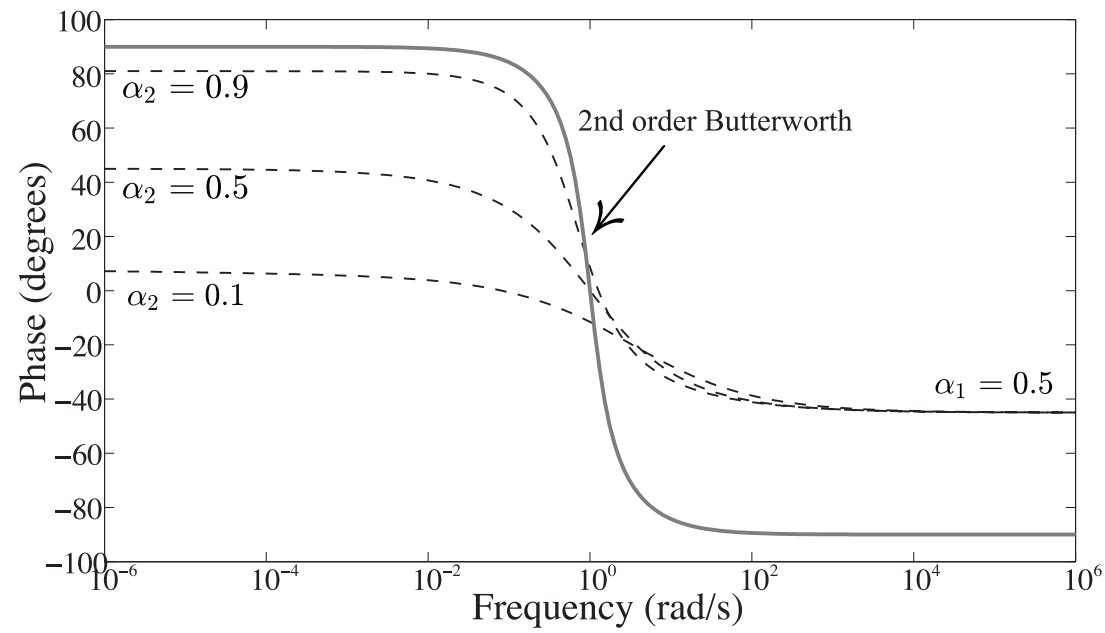

(b)

Fig. 5. Magnitude and phase responses of FBPFs of order $\left(\alpha_{1}+\alpha_{2}\right)=0.6$, 1.0 and 1.4 when $\alpha_{1}=0.5$ and $a=b=c=1$.

with the values of $x_{n}$, where $n \in[0,5]$, given in Table II. The MATLAB simulated normalized magnitude and phase responses of (6) with fractional orders of $\left(\alpha_{1}+\alpha_{2}\right)=0.6,1.0$, and 1.4 when $\alpha_{1}=0.5$ and $a=b=c=1$ compared to the $2^{\text {nd }}$ Butterworth BP response with centre frequency $\omega_{0}=1 \mathrm{rad} / \mathrm{s}$ and bandwidth $B=1$, labelled 2nd order Butterworth, are illustrated in Figs. 5(a) and 5 (b), respectively. From the simulated magnitude responses, we see that the low frequency stopband has attenuations of $-1.55,-10$, and $-18 \mathrm{~dB} /$ decade while the high frequency stopband maintains an attenuation of $-10 \mathrm{~dB} /$ decade. The stopband attenuations very closely match those predicted and confirm that the low and high frequency stopband attenuations are independent of each other. We also note that the fractional step phases of $-9^{\circ},-45^{\circ}$ and $-81^{\circ}$ when $\alpha_{2}=0.1,0.5$, and 0.9 , respectively, match the expected phase, $-90 \alpha_{2}^{\circ}$, predicted by (11) when $\omega=0$. As well, the high frequency phases of $-45^{\circ}$ when $\alpha_{1}=0.5$ match the $-90 \alpha_{1}^{\circ}$ predicted by (11) when $\omega=\infty$. In Figs. 6(a) and 6(b), MATLAB simulated normalized magnitude and phase responses of (6), with fractional stepping in the high frequency stopband, with orders of $\left(\alpha_{1}+\alpha_{2}\right)=0.6,1.0$ and 1.4 when $\alpha_{2}=0.5$ and $a=b=c=1$ compared to the $2^{\text {nd }}$-order bandpass transformed Butterworth response, when $\omega_{0}=B=1$, are illustrated. Again, we see the high frequency stopband has attenuations of $-1.55,-10$, and $-18 \mathrm{~dB} /$ decade while the low frequency stopband maintains an attenuation of -10 $\mathrm{dB} /$ decade, very closely matching those predicted. We also note the high frequency fractional step of the phase response matches the predicted $-90 \alpha_{1}{ }^{\circ}$ and the low frequency phase of $-45^{\circ}$ matches that predicted by $-90 \alpha_{2}^{\circ}$. 


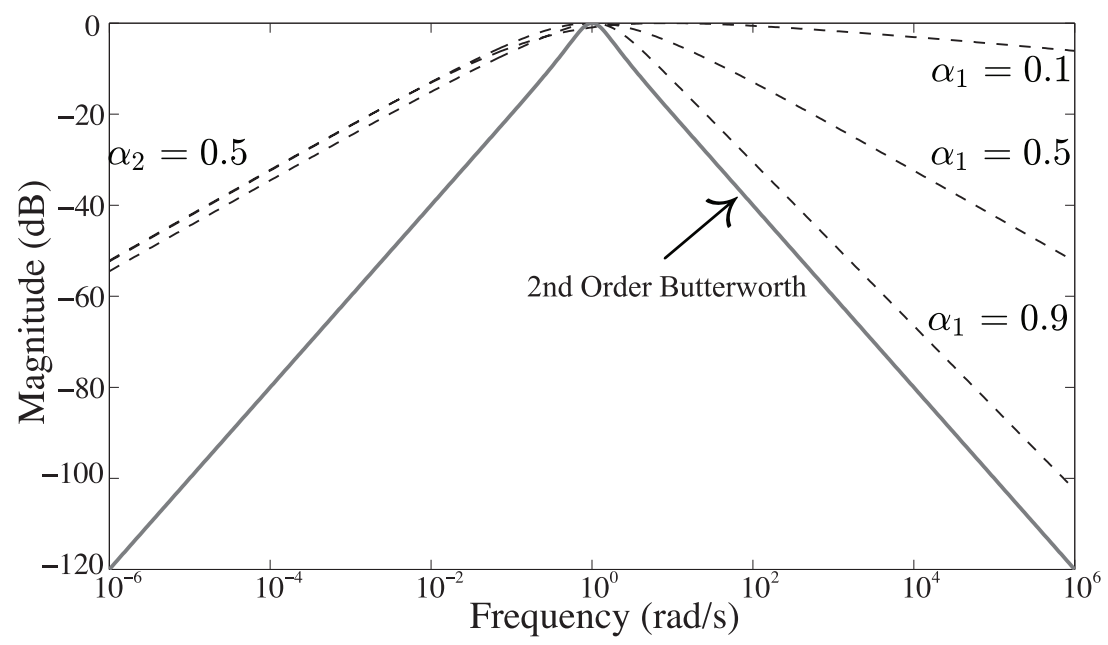

(a)

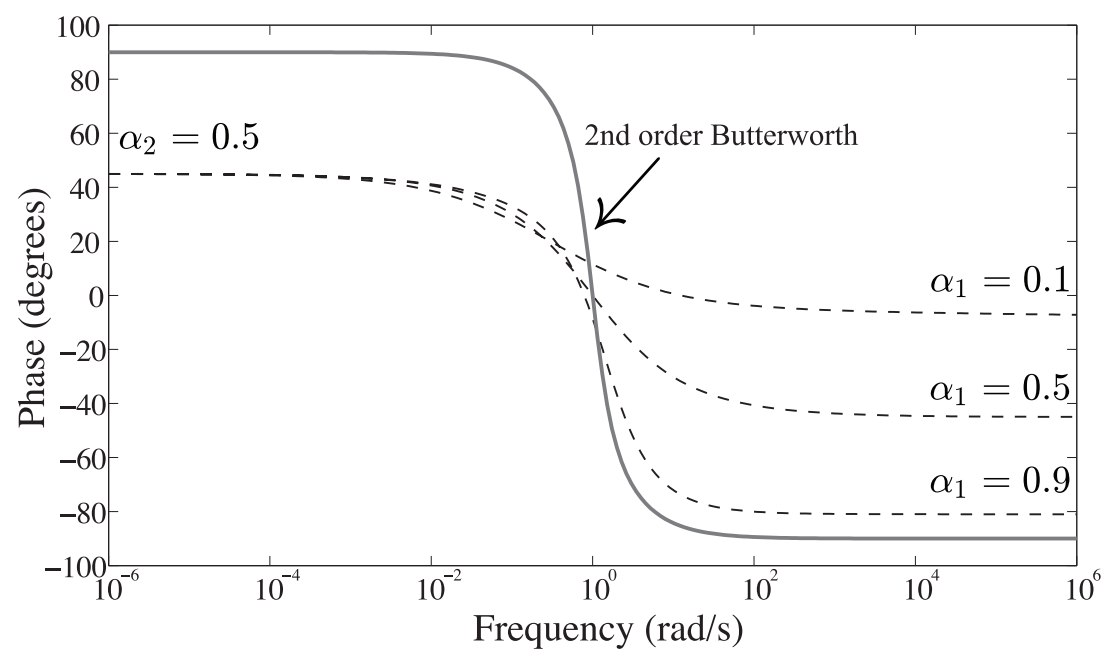

(b)

Fig. 6. Magnitude and phase responses of FBPFs of order $\left(\alpha_{1}+\alpha_{2}\right)=0.6$, 1.0 and 1.4 when $\alpha_{2}=0.5$ and $a=b=c=1$.

From the magnitude responses of Fig. 5(a) and 6(a) the frequency location at which the magnitude has a maxima is not located at $1 \mathrm{rad} / \mathrm{s}$ as is the case for the Butterworth response. The location of the FBPF maxima frequency, $\omega_{m}$, can be obtained by solving the equation

$$
\begin{aligned}
0= & x^{2 \alpha_{2}+\alpha_{1}} a c\left(\alpha_{2}-\alpha_{1}\right) \cos \left(\frac{\left(\alpha_{1}+\alpha_{2}\right) \pi}{2}\right)+x^{\alpha_{2}} a c \alpha_{2}\left(x^{\alpha_{2}} \cos \left(\frac{\alpha_{2} \pi}{2}\right)+c\right) \\
& -x^{3 \alpha_{2}+\alpha_{1}} a \alpha_{1}\left(x^{\alpha_{1}}+b \cos \left(\frac{\alpha_{1} \pi}{2}\right)\right)
\end{aligned}
$$

for its positive real root where $x=\omega_{m}$. The maxima frequencies numerically calculated using (12) when $0<\alpha_{1,2} \leq 1$ and $a=b=c=1$ are given in Fig. 7. We note from Fig. 7 when $\alpha_{1}=\alpha_{2}$ that $\omega_{m}=1 \mathrm{rad} / \mathrm{s}$ as expected. Also, the calculated maximum frequencies show a reciprocal mirroring along the line $\alpha_{1}=\alpha_{2}$ such that

$$
\left.\omega_{m}\right|_{\alpha_{1}=i, \alpha_{2}=j}=\left.\frac{1}{\omega_{m}}\right|_{\alpha_{1}=j, \alpha_{2}=i}
$$

for pairs of points where $0<i, j \leq 1$. The most extreme case of this symmetry in Fig. 7 occurs with the pair $\left(\alpha_{1}, \alpha_{2}\right)=(0.1,0.3)$ and $(0.3,0.1)$ corresponding to maxima frequencies $\omega_{m}=12.15 \mathrm{rad} / \mathrm{s}$ and $0.0823 \mathrm{rad} / \mathrm{s}$, respectively, where $12.15=\frac{1}{0.0823}$ as predicted by (13).

These simulations highlight that the fractional Tow-Thomas biquad is able to realize bandpass filters with independent asymmetric stopband characteristics, with the fractional orders of $C_{1}$ and 


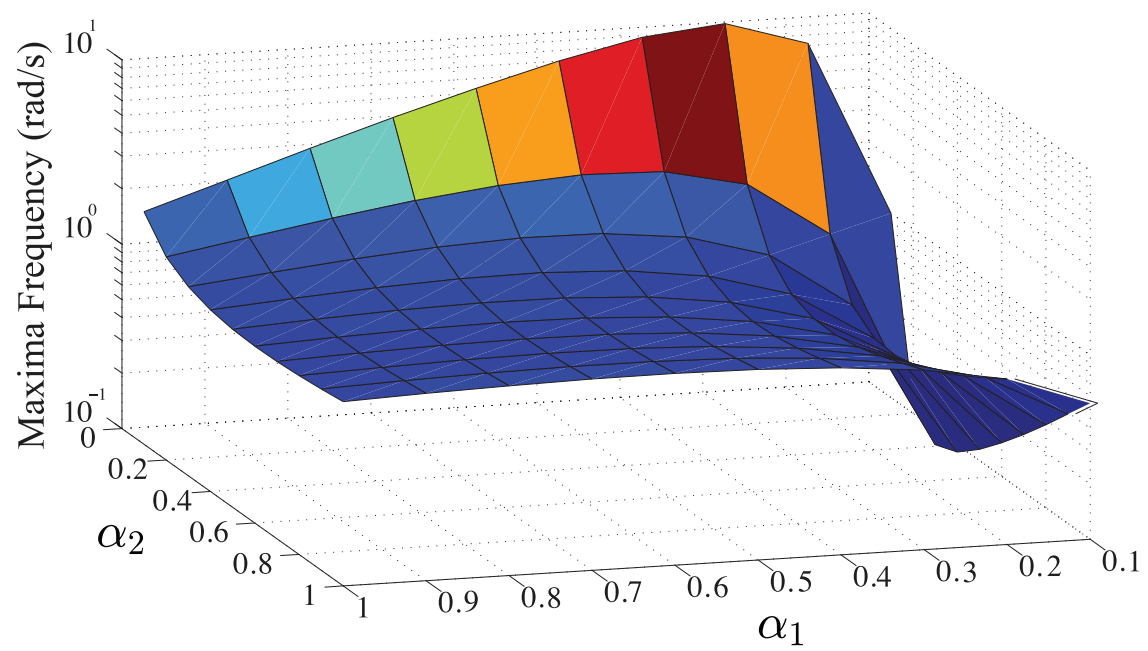

Fig. 7. Calculated maxima frequencies of FBPFs using (12) when $0<\alpha_{1,2} \leq$ 1 in steps of 0.1 and $a=b=c=1$.

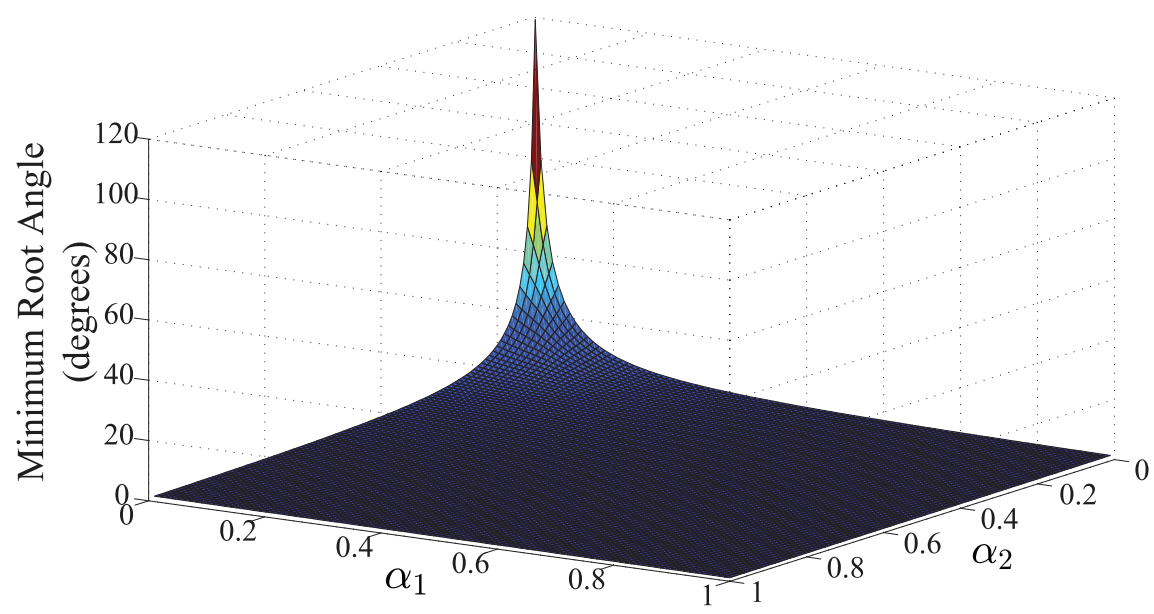

Fig. 8. Minimum root angle in the $W$-plane of (14) for $0<\alpha_{1} \leq 1$ and $0<\alpha_{2} \leq 1$ when $b=c=1$.

$C_{2}$ controlling the attenuation of the high or low frequency stopbands, respectively. It is worth noting that designing asymmetric BPFs is not easily done with classical design techniques but in fact is difficult and challenging [21,22]. The fractional Tow-Thomas biquad provides control of the attenuation specifications of each stopband through manipulation of only a single design parameter which was also not possible using the method in [16].

\subsection{Stability of fractional Tow-Thomas biquad}

To analyze the stability of the fractional step lowpass and bandpass filter responses of the fractional Tow-Thomas biquad, we transform the transfer function from the $s$-plane to the $W$-plane using the transformations $s=W^{m}$ and $\alpha_{n}=k_{n} / m$ [23]. This results in transforming the transfer function from a fractional to an integer order one which can be analyzed using traditional integer order analysis methods. Applying the transformation to the denominator of (5) and (6) yields:

$$
s^{\alpha_{1}+\alpha_{2}}+b s^{\alpha_{2}}+c=0 \rightarrow W^{k_{1}+k_{2}}+b W^{k_{2}}+c=0
$$

which is the characteristic equation in the $W$-plane that must be solved to ensure it meets the stability criteria. This stability criteria in the $W$-plane requires $\left|\theta_{W}\right|>\frac{\pi}{2 m}$ for all poles of the characteristic equation, where $\theta_{W}$ is the pole angle in radians. If any pole angle does not meet this criteria then the system is unstable. Therefore, knowing $b, c, \alpha_{1}$, and $\alpha_{2}$ for the desired filter response we can calculate the pole angles to check for stability. The minimum root angles in the $W$-plane of (14) for $0<\alpha_{1} \leq 1$ and $0<\alpha_{2} \leq 1$ when $b=c=1$ are shown in Fig. 8. For this analysis, $\alpha_{1}$ and $\alpha_{2}$ were 
both incremented in steps of 0.01 , corresponding to $m=100$, which requires $\left|\theta_{W}\right|>0.9^{\circ}$ for stability. From this figure we observe that (5) and (6) are stable for all $0<\alpha_{1} \leq 1$ and $0<\alpha_{2} \leq 1$ when $b=c=1$, with the phase margin decreasing as both $\alpha_{1}$ and $\alpha_{2}$ increase, reaching a minimum pole angle of $1.2^{\circ}$ when $\alpha_{1}=\alpha_{2}=1$. Therefore, all of the simulated fractional lowpass and bandpass filter responses in Sections 2.1 and 2.2, respectively, are stable ${ }^{1}$.

Solving (14) in the $s$-domain when $\alpha_{1}=\alpha_{2}=1$ and $b=c=1$, the minimum pole angle case in the $W$-plane, yields two poles at $s=1 \measuredangle \pm 120^{\circ}$ with a stability margin of $30^{\circ}$. Knowing that $s=W^{m}$ the corresponding poles in the $W$-plane become

$$
\begin{aligned}
W & =1 \measuredangle \frac{ \pm 120^{\circ}}{m} \\
& =1 \measuredangle \pm 1.2^{\circ}
\end{aligned}
$$

Showing that the pole angles of $\pm 1.2^{\circ}$ in the $W$-plane, when $m=100$, correspond to $\pm 120^{\circ}$ in the $s$-plane. Therefore, the fractional-order Tow-Thomas will always have a better stability margin than the $2^{\text {nd }}$ order.

\section{Low pass filter without passband peaking}

The transfer function (5), realized by the fractional Tow-Thomas biquad, can also be used to implement an FLPF without the passband peaking problem, as proposed in [20]. In this case, $\alpha_{1}=1$ and the transfer function has the form

$$
H_{1+\alpha}^{L P}(s)=\frac{a}{s^{1+\alpha_{2}}+b s^{\alpha_{2}}+c}
$$

which was shown to be stable for all $a, b$, and $c$ values. Comparing the coefficients of (3) to (15) while ignoring the sign in (3) yields the relationships given in Table I. Using the values of $a, b$, and $c$ in Table I for the FLPF we have 3 design equations and 8 variables yielding 5 degrees of freedom in our selection of the component values required to realize the desired $a, b$, and $c$ values in the fractional low pass filter. Therefore, setting $C_{1}=C_{2}=1 \mathrm{~F}$ and $R_{2}=R_{4}=R_{5}=1 \Omega$ our design equations for the remaining components become

$$
\begin{aligned}
R_{1} & =\frac{c}{a} \frac{R_{2} R_{5}}{R_{4}}=\frac{c}{a} \\
R_{3} & =\frac{1}{b} \frac{1}{C_{1}}=\frac{1}{b} \\
R_{6} & =c C_{1} C_{2} R_{2} R_{5}^{2}=c
\end{aligned}
$$

Using Eqs. (16) to (18) we can design a FLPF free from the passband peaking problem by selecting $b$ and $c$ such that

$$
\begin{aligned}
& b=1.1796 \alpha_{2}^{2}+0.16765 \alpha_{2}+0.21735 \\
& c=0.19295 \alpha_{2}+0.81369
\end{aligned}
$$

where $b$ and $c$ were calculated for the passband of (5) to resemble that of the first order Butterworth response [20]. This response can be scaled to a desired frequency through the application of the frequency and magnitude scaling factors

$$
\begin{aligned}
C_{\text {new }} & =\frac{C_{\text {old }}}{K_{f} K_{m}} \\
R_{\text {new }} & =R_{\text {old }} K_{m}
\end{aligned}
$$

where $K_{m}$ is the desired magnitude scaling factor, $K_{f}=\omega^{\alpha}$ is the frequency scaling factor [12], and $\omega$ is the desired frequency to be shifted to. The element values required to realize the transfer function (15) magnitude scaled by a factor of 1000 and frequency shifted to $1 \mathrm{kHz}$ when $\alpha_{2}=0.1,0.5$, and $0.9, a=1$ and $b$ and $c$ are selected for minimum passband error are given in Table III.

\footnotetext{
${ }^{1}$ The cases where $b \neq c=1$ can still be stable but need to by analyzed on a case-by-case basis. An analysis of the minimum values of $b$ required for stability with fixed $c$ for $1+\alpha$ order filters when $0 \leq \alpha \leq 1$ is given in [20].
} 
Table III. Component values to realize (15) when $\alpha_{2}=0.1,0.5$, and 0.9 , $\alpha_{1}=a=1$ and $b$ and $c$ are selected for minimum passband error.

\begin{tabular}{|c|c|c|c|}
\hline \multirow{2}{*}{ Component } & \multicolumn{3}{|c|}{ Values for FLPF of order } \\
\cline { 2 - 4 } & 1.1 & 1.5 & 1.9 \\
\hline \hline$C_{1}(\mu \mathrm{F})$ & \multicolumn{3}{|c|}{0.159} \\
\hline$C_{2}(\mu \mathrm{F})$ & 417 & 12.6 & 0.382 \\
\hline$R_{1}(\Omega)$ & 833 & 910 & 987 \\
\hline$R_{3}(\Omega)$ & 4067 & 1678 & 755 \\
\hline$R_{6}(\Omega)$ & 833 & 910 & 987 \\
\hline$R_{2}, R_{4}, R_{5}(\Omega)$ & \multicolumn{4}{|c|}{1000} \\
\hline
\end{tabular}

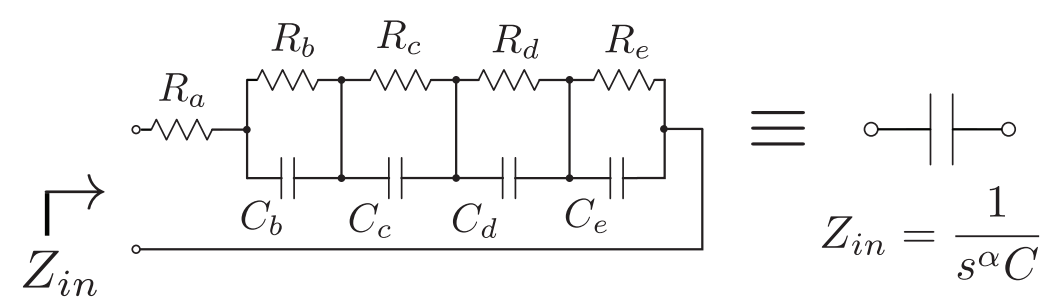

Fig. 9. RC ladder network to realize a $4^{\text {th }}$ order approximated fractional capacitor.

Table IV. Component values to realize $4^{\text {th }}$ order approximations of fractional capacitors with values of $417 \mu \mathrm{F}, 12.6 \mu \mathrm{F}, 0.382 \mu \mathrm{F}$ and orders of $0.1,0.5$, and 0.9 , respectively. The center frequency is $1 \mathrm{kHz}$.

\begin{tabular}{|c|c|c|c|}
\hline \multirow{2}{*}{ Component } & \multicolumn{3}{|c|}{ Values } \\
\cline { 2 - 4 } & $C_{2}=417 \mu \mathrm{F}$ & $C_{2}=12.6 \mu \mathrm{F}$ & $C_{2}=0.382 \mu \mathrm{F}$ \\
\cline { 2 - 4 } & $\alpha_{2}=0.1$ & $\alpha_{2}=0.5$ & $\alpha_{2}=0.9$ \\
\hline \hline$R_{a}(\Omega)$ & 658.7 & 111.1 & 6.8 \\
\hline$R_{b}(\Omega)$ & 196.3 & 251.7 & 43.3 \\
\hline$R_{c}(\Omega)$ & 134.6 & 378.7 & 130.7 \\
\hline$R_{d}(\Omega)$ & 159.0 & 888.9 & 670.4 \\
\hline$R_{e}(\Omega)$ & 369.5 & 7369.7 & 146189.7 \\
\hline$C_{b}(\mathrm{nF})$ & 68.9 & 83.8 & 705 \\
\hline$C_{c}(\mu \mathrm{F})$ & 0.627 & 0.296 & 1.13 \\
\hline$C_{d}(\mu \mathrm{F})$ & 2.18 & 0.537 & 1.03 \\
\hline$C_{e}(\mu \mathrm{F})$ & 6.64 & 0.695 & 0.207 \\
\hline
\end{tabular}

\subsection{PSPICE simulations}

While most capacitors do exhibit fractional behaviour [3,4] and should be modeled with an impedance $Z_{C}=\frac{1}{s^{\alpha} C}$, the value of $\alpha$ is very near to 1 preventing their use in implementing fractional filters. Therefore, until commercial fractance devices become available to physically realize circuits that make use of $s^{\alpha}$, integer order approximations have to be used. There are many methods to create an approximation of $s^{\alpha}$ that include Continued Fraction Expansions (CFEs) as well as rational approximation methods [5]. These methods present a large array of approximations with varying order and accuracy, with the accuracy and approximated frequency band increasing as the order of the approximation increases. Here, a CFE method [24] was selected to model the fractional capacitors for PSPICE simulations. Collecting eight terms of the CFE yields a $4^{\text {th }}$ order approximation of the fractional capacitor that can be physically realized using the RC ladder network in Fig. 9.

The component values required for the $4^{\text {th }}$ order approximation of $C_{2}$ with values from Table III and orders of $0.1,0.5$, and 0.9 using the RC ladder network in Fig. 9, shifted to a center frequency of $1 \mathrm{kHz}$, are given in Table IV. The magnitude and phase of the ideal (solid line) and $4^{\text {th }}$ order approximated (dashed) fractional capacitor with capacitance $12.6 \mu \mathrm{F}$ and order $\alpha=0.5$, shifted to a center frequency of $1 \mathrm{kHz}$, are presented in Fig. 10(a). From this figure we observe that the approximation is very 


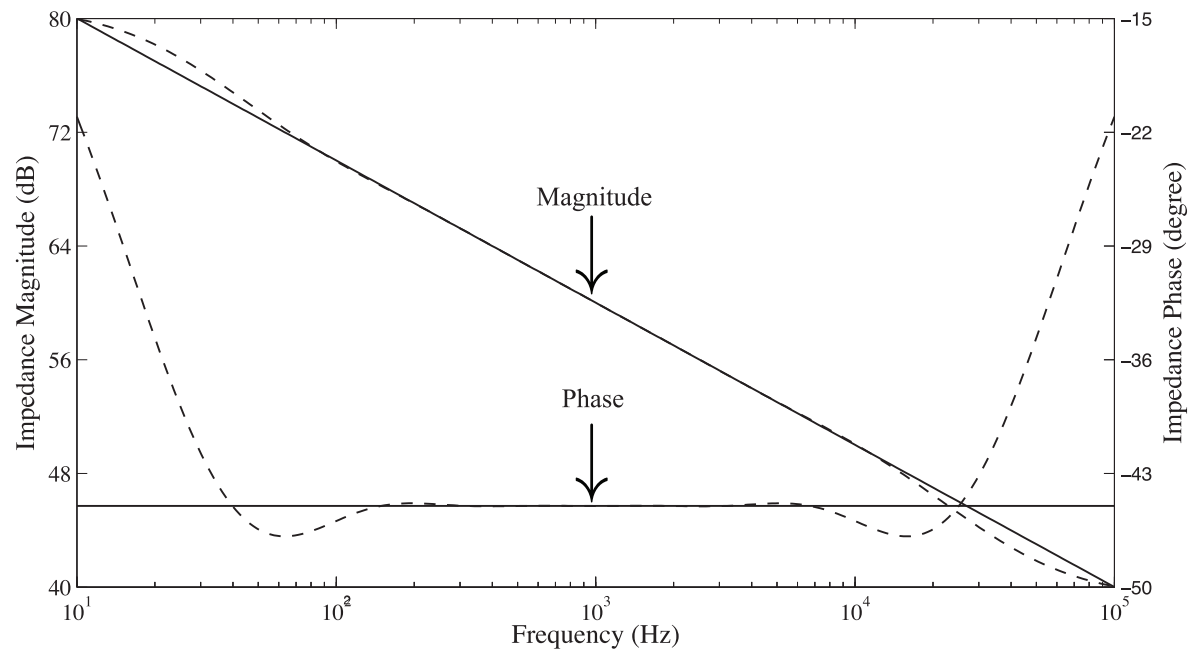

Fig. 10. Magnitude and phase response of the approximated fractional capacitor (dashed) compared to the ideal (solid) with capacitance of $12.6 \mu \mathrm{F}$ and order 0.5 after scaling to a center frequency of $1 \mathrm{kHz}$.

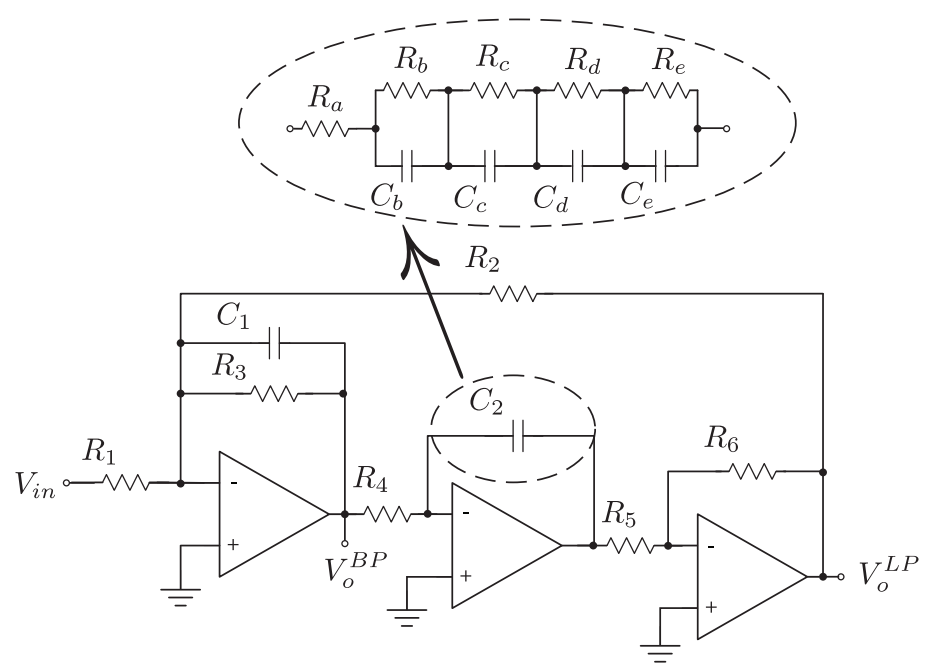

Fig. 11. Fractional Tow-Thomas biquad with the RC ladder network to realize a $4^{\text {th }}$ order approximation of the fractional capacitor $C_{2}$.

good over almost 4 decades, from $200 \mathrm{~Hz}$ to $70 \mathrm{kHz}$, for the magnitude and almost 2 decades, from $200 \mathrm{~Hz}$ to $6 \mathrm{kHz}$, for the phase. In these regions, the deviation of the approximation from ideal does not exceed $1.23 \mathrm{~dB}$ and $0.23^{\circ}$ for the magnitude and phase, respectively. Using the component values in Tables III and IV, the approximated fractional Tow-Thomas biquad, shown in Fig. 11, was simulated using MC1458 op amps to realize fractional lowpass and bandpass filters of orders $\left(\alpha_{1}+\alpha_{2}\right)=1.1,1.5$, and 1.9 when $\alpha_{1}=1^{2}$ and $\alpha_{2}=0.1,0.5$, and 0.9. The simulated magnitude and phase responses (dashed lines) at the lowpass node compared to the ideal response (solid lines) are shown in Figs. 12(a) and (b), respectively. We can clearly see from the magnitude response of both the MATLAB and PSPICE simulated responses that this filter does realize a fractional step through the stopband of the filter. Note though, that the PSPICE simulated stopband deviated from the MATLAB simulated transfer function; which is a result of using the $4^{\text {th }}$ order approximation of $C_{2}$. This effect is much more pronounced in the phase response than the magnitude response because the phase approximations deviate from the ideal at $6 \mathrm{kHz}$, much lower than the $70 \mathrm{kHz}$ deviation location for the magnitude approximation. Comparing the results, we find that the stopband attenuation of the simulated filters are very close to their theoretical values of $-20\left(1+\alpha_{2}\right) \mathrm{dB} /$ decade. For comparison, these attenuations are listed in Table V. Also, the simulated magnitude and phase responses (dashed

${ }^{2}$ Note that $\alpha_{1}=1$ means that $C_{1}$ is a normal capacitor 


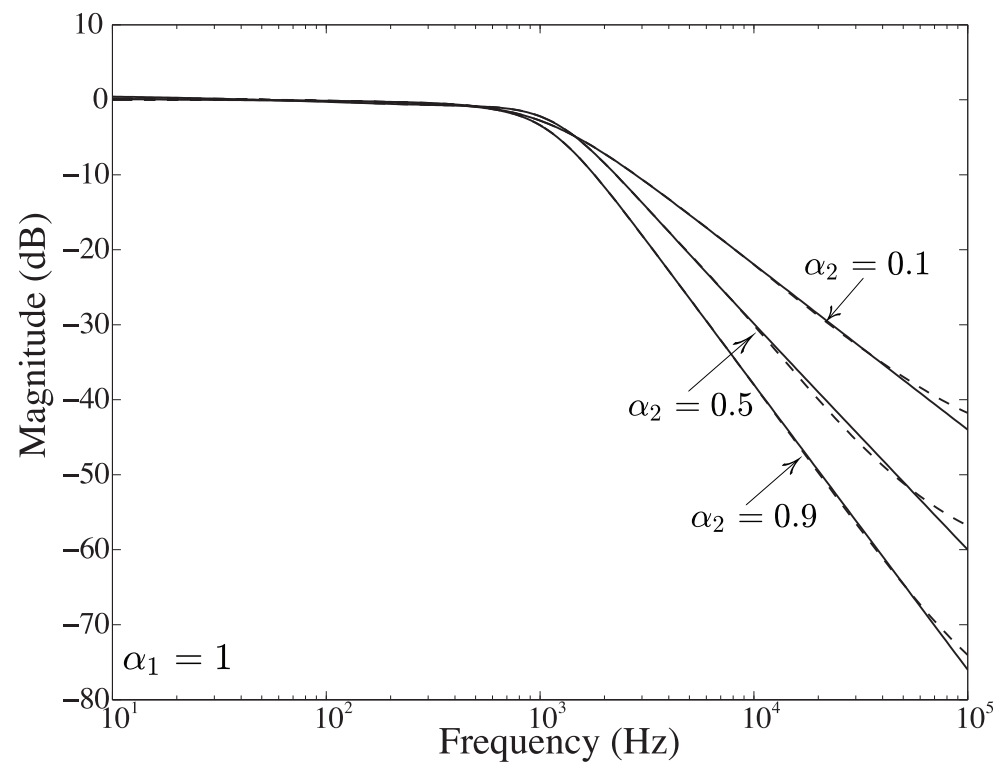

(a)

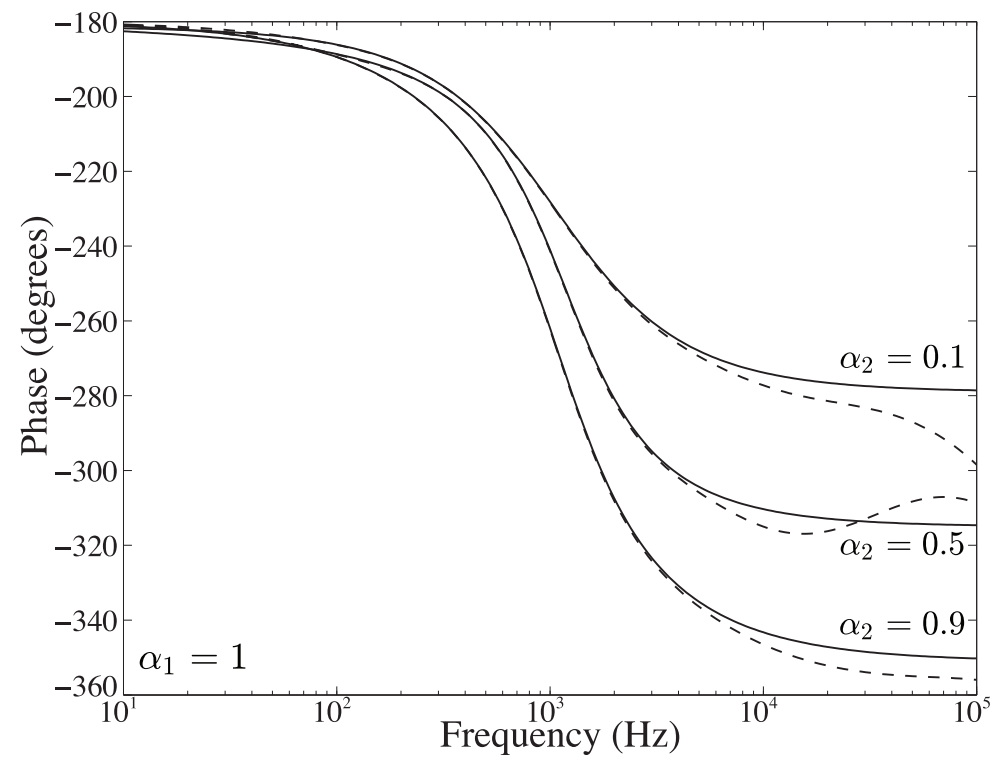

(b)

Fig. 12. MATLAB (solid) and PSPICE (dashed) simulation results of the (a) magnitude and (b) phase response of the approximated $\left(1+\alpha_{2}\right)$ order FLPFs.

Table V. Theoretical, MATLAB, and PSPICE simulated stopband attenuations of approximated $\left(1+\alpha_{2}\right)$ order FLPF.

\begin{tabular}{|c|c|c|c|}
\hline Order & \multicolumn{3}{|c|}{ Stopband Attenuation (dB/decade) } \\
\cline { 2 - 4 }$\left(1+\alpha_{2}\right)$ & Theoretical & MATLAB & PSPICE \\
\hline \hline 1.1 & -22 & -22.57 & -21.81 \\
\hline 1.5 & -30 & -30.05 & -29.82 \\
\hline 1.9 & -38 & -38.01 & -38.09 \\
\hline
\end{tabular}

lines) at the bandpass node compared to the ideal response (solid lines) show very good agreement and are given in Figs. 13(a) and 13(b) confirming that the fractional Tow-Thomas biquad can realize asymmetric fractional bandpass filter characteristics when $\alpha_{1} \neq \alpha_{2}$. The low and high frequency stopband attenuations are very close to their theoretical values of $-20 \alpha_{2} \mathrm{~dB} /$ decade and $-20 \alpha_{1}$ $\mathrm{dB} /$ decade, respectively, with their measured MATLAB and PSPICE values given in Table VI. 


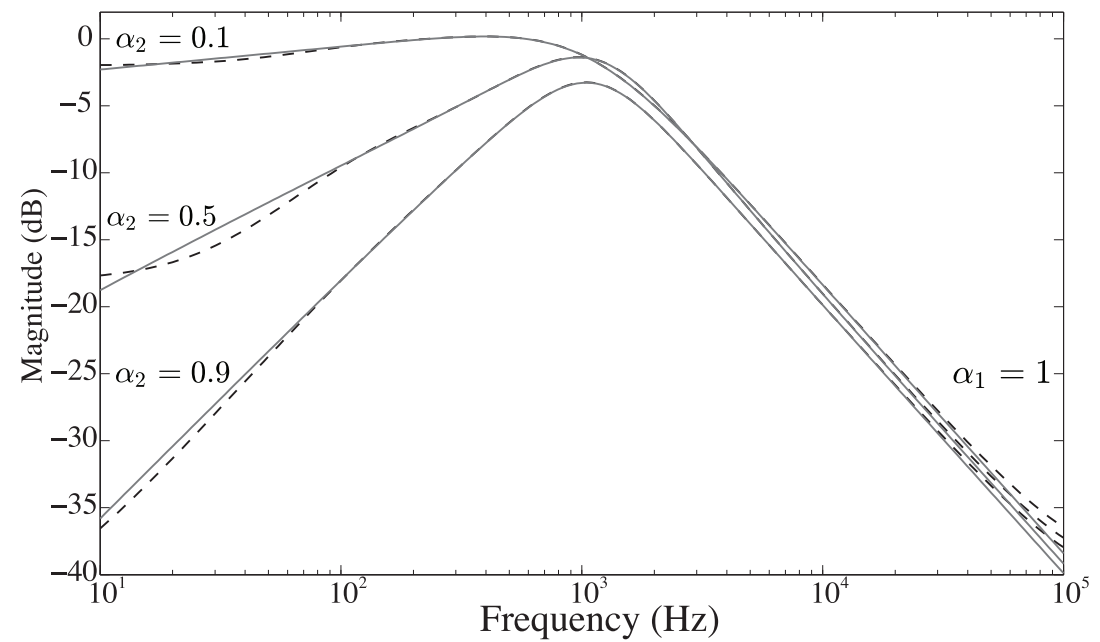

(a)

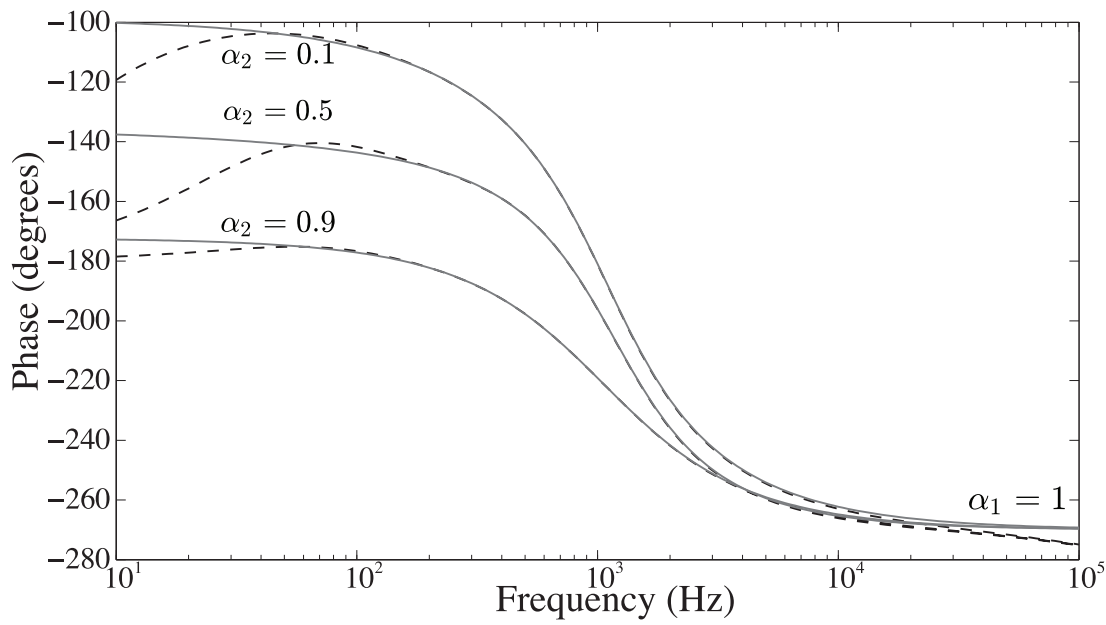

(b)

Fig. 13. MATLAB (solid) and PSPICE (dashed) simulation results of the (a) magnitude and (b) phase response of the approximated $\left(1+\alpha_{2}\right)$ order FBPFs.

Table VI. Theoretical, MATLAB, and PSPICE simulated low and high frequency stopband attenuations of approximated $\left(1+\alpha_{2}\right)$ order FBPF.

\begin{tabular}{|c|c|c|c|c|c|c|}
\hline Order & \multicolumn{2}{|c|}{ Low Frequency Attenuation (dB/decade) } & \multicolumn{3}{c|}{ High Frequency Attenuation (dB/decade) } \\
\hline$\left(1+\alpha_{2}\right)$ & Theoretical & MATLAB & PSPICE & Theoretical & MATLAB & PSPICE \\
\hline \hline 1.1 & -2 & -1.71 & -1.73 & -20 & -20.06 & -19.56 \\
\hline 1.5 & -10 & -9.27 & -9.75 & -20 & -20.66 & -19.76 \\
\hline 1.9 & -18 & -17.85 & -18.49 & -20 & -20.08 & -19.88 \\
\hline
\end{tabular}

\section{Experimental results}

While there are currently no commercial fractional capacitors available for the physical implementation of fractional order circuits, there are reported prototype implementations of such devices [6,25-28] using fractal shapes. A fractal is "a rough or fragmented geometric shape that can be split into parts, each of which is (at least approximately) a reduced-sized copy of the whole" [29]. Fractals such as the Sierpinski [30], Tree [25, 27], Hilbert [6], Koch and Peano [28] structures, when implemented as one of the plates in a capacitor, have been shown to exhibit fractional order electrical impedances. These fractional capacitors (FCs) can be used in the realization of fractional systems without having to use an integer order approximation of the FCs. Two such devices [26] were used to realize the fractional Tow-Thomas biquad of Fig. 1. These two fractional capacitors are shown in Fig. 14(a), labelled as Hilbert and Arbre, with the fractal structures after which they were named shown in Fig. 14(b) and 


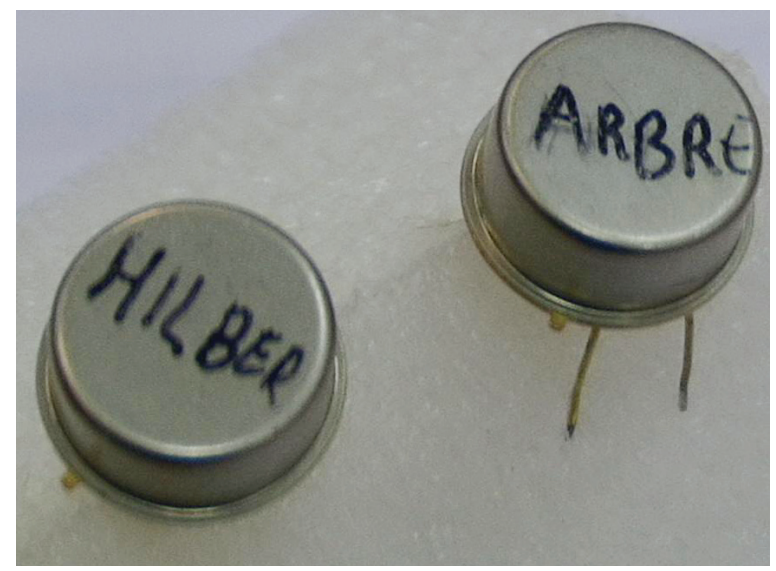

(a)

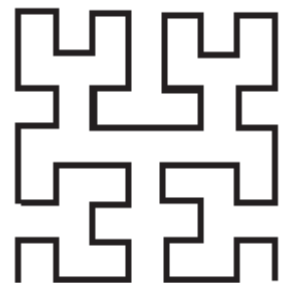

(b)

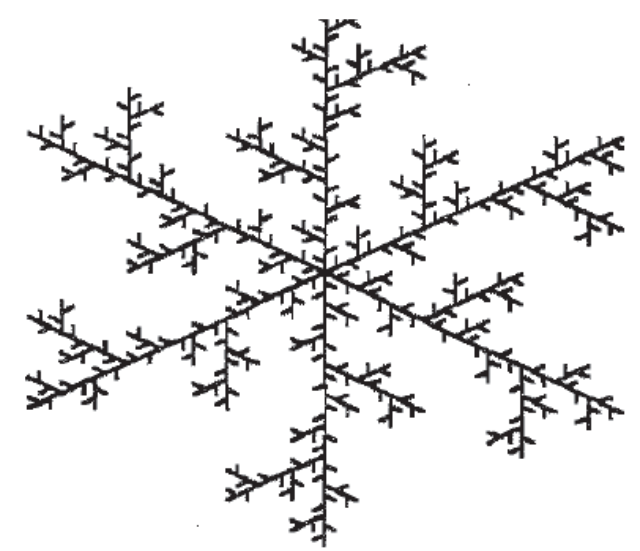

(c)

Fig. 14. (a) Packaged fractional capacitors implemented using photolithographic fractal structures on silicon, based on the (b) Hilbert and (c) Tree fractal structures.

Table VII. Component values to realize $2^{\text {nd }}$ order Butterworth LP response with cutoff frequency of $500 \mathrm{~Hz}$ using the Tow-Thomas Biquad.

\begin{tabular}{|c|c|}
\hline Component & Value \\
\hline \hline$C_{1}, C_{2}$ & $6.8 \mathrm{nF}$ \\
\hline$R_{1}, R_{2}, R_{4}, R_{5}, R_{6}$ & $46.8 \mathrm{k} \Omega$ \\
\hline$R_{3}$ & $33.1 \mathrm{k} \Omega$ \\
\hline
\end{tabular}

(c), respectively.

Both the Hilbert and Arbre fractional capacitors, with fractional orders of 0.5 [26], were used to implement Fig. 1 with quality factor $Q=1 / \sqrt{2}$ and cutoff frequency $\mathrm{f}_{o}=500 \mathrm{~Hz}$. The component values required for this realization are given in Table VII. This circuit was breadboarded using MC1458 op amps with $5 \%$ and $20 \%$ tolerance resistors and capacitors, respectively. The magnitude response at the low-pass output node of the circuit was measured using a HP4395A Network analyzer. For comparison, MATLAB simulations of (3) and experimental results of the Tow-Thomas biquad 


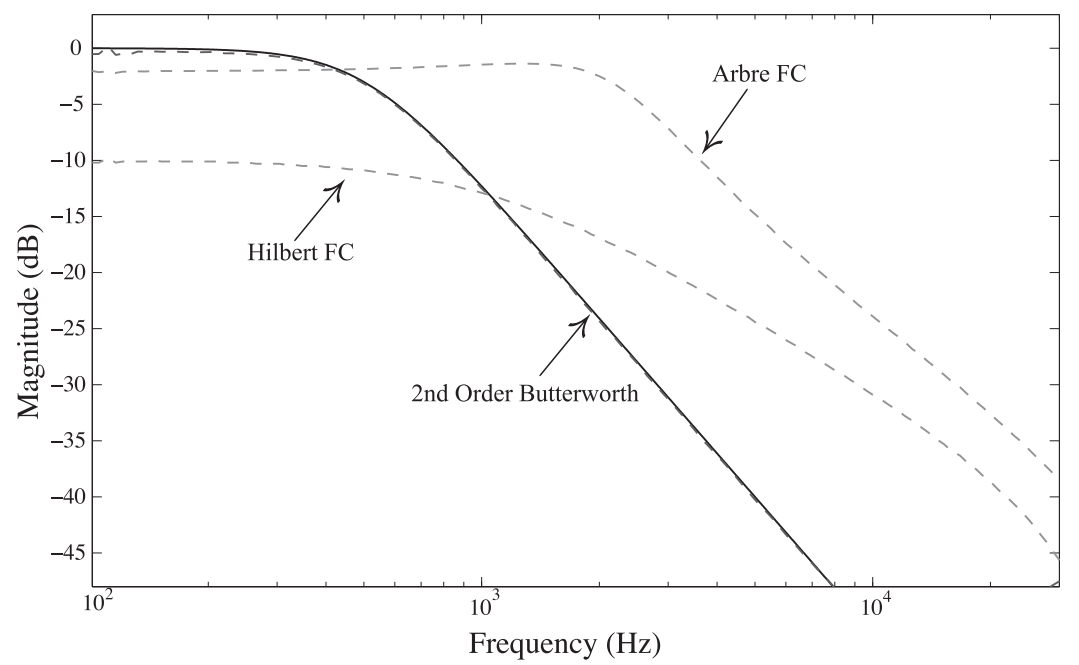

Fig. 15. Experimental magnitude responses of fractional Tow-Thomas using traditional capacitor (solid) and Hilbert and Arbre fractional capacitors (dashed).

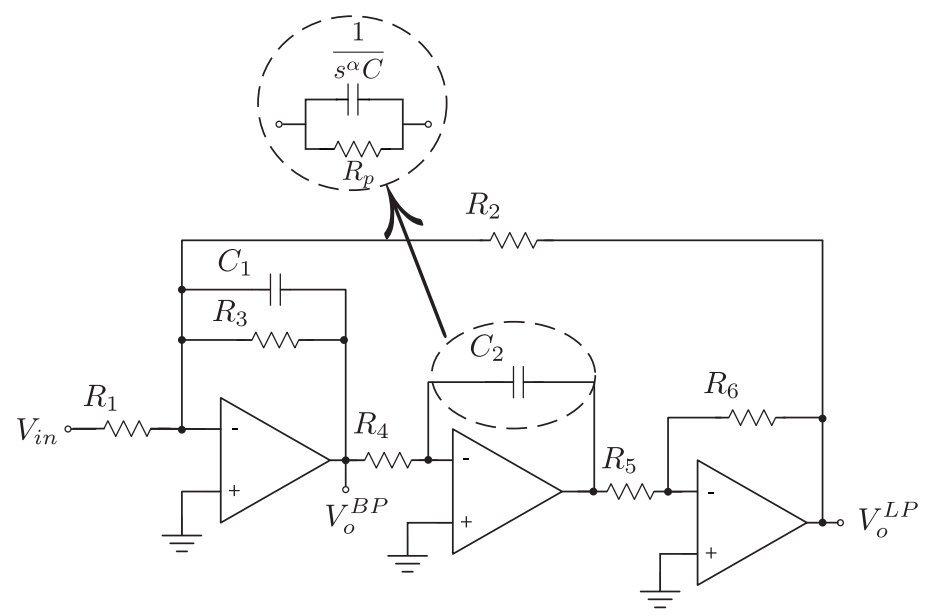

Fig. 16. Tow-Thomas biquad circuit using parallel resistance impedance model of Hilbert FC.

designed to realize a $2^{\text {nd }}$ order low pass Butterworth response are plotted in Fig. 15 as solid and dashed lines, respectively. The experimental magnitude responses at the lowpass node when $C_{2}$ was replaced by the the Hilbert and Arbre fractional capacitors are also given in Fig. 15. We clearly observe the fractional behaviour in the stopband of the response using the Arbre FC, with an attenuation of $-31.22 \mathrm{~dB} /$ decade, very closely matching the $-20\left(\alpha_{1}+\alpha_{2}\right)=-30 \mathrm{~dB} /$ decade expected from (5) when $\alpha_{1}=1$ and $\alpha_{2}=0.5$. However, the stopband attenuation of the response using the Hilbert FC does not match the theoretical expectation. Further investigation into the Hilbert FC indicated that it has a resistance in parallel with its fractional capacitor component, the impedance model based on this parallel resistance is shown in the inset of Fig. 16. The transfer function of the fractional Tow-Thomas biquad at the LP output node, in this case, becomes

$$
\frac{V_{o}^{L P}(s)}{V_{i n}(s)}=-\frac{\frac{R_{5}}{R_{1} R_{4} R_{6} C_{1} C_{2}}}{s^{1+\alpha}+s \frac{1}{R_{p} C_{2}}+s^{\alpha} \frac{1}{R_{3} C_{1}}+\frac{R_{5}\left(R_{2} R_{4}+R_{3} R_{p}\right)}{R_{2} R_{3} R_{4} R_{6} R_{p} C_{1} C_{2}}}
$$

Note that when (21) is compared to (5), there is an extra term in the denominator and that the DC gain changes which explains the significant change in the low frequency gain and stopband attenuation of the experimental results using the Hilbert FC.

Using the transfer functions of (15) and (21), a non-linear least squares method was used to extract the impedance parameters of the Arbre and Hilbert fractional capacitors, respectively, from the experimentally collected frequency response. This numerical method attempts to solve the problem 
Table VIII. Impedance parameters of Hilbert and Arbre fractional capacitors extracted from experimental results using non-linear least squares fitting.

\begin{tabular}{|c|c|c|c|}
\hline Fractional Capacitor & $\alpha$ & $C(\mathrm{~F})$ & $R_{p}(\mathrm{k} \Omega)$ \\
\hline \hline Arbre & 0.4634 & $0.101 \mu$ & $\infty$ \\
\hline Hilbert & 0.7589 & $2.98 \mathrm{n}$ & 30.437 \\
\hline
\end{tabular}

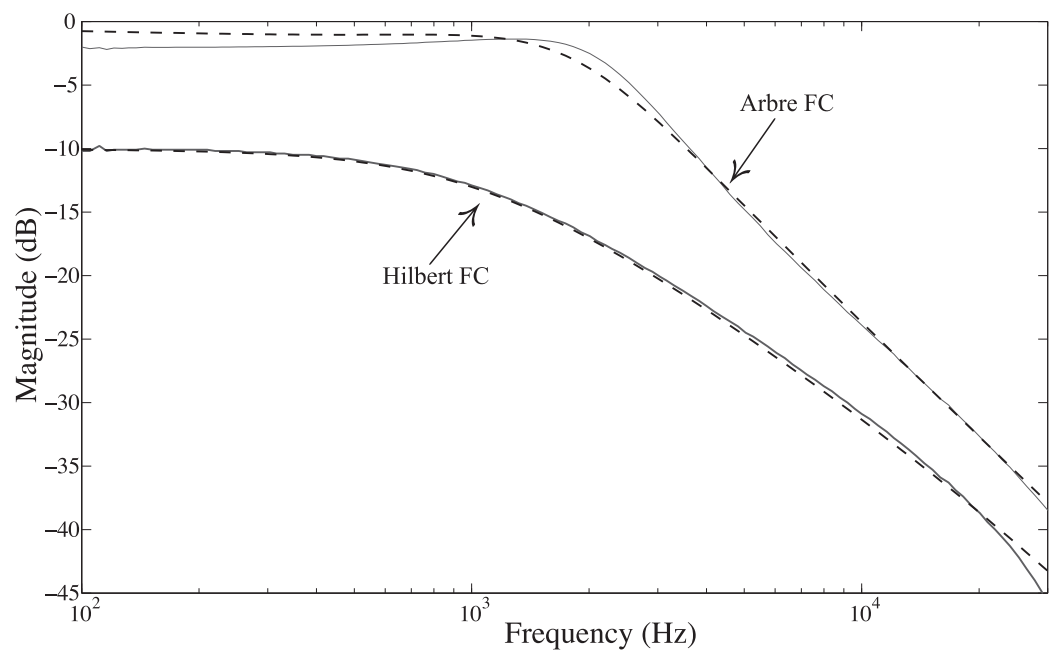

Fig. 17. MATLAB simulation of (15) and (21) using least squares parameters (solid) compared to experimental (dashed) magnitude responses of fractional Tow-Thomas biquad using Hilbert and Arbre fractional capacitors.

$$
\min _{x}\|T(x)-y d a t a\|_{2}^{2}=\min _{x} \sum_{i}^{n}\left(T(x)_{i}-y_{d a t a}\right)^{2}
$$

where $x$ is the vector of impedance parameters $\left(\alpha_{2}, C_{2}\right)$ and $\left(\alpha_{2}, C_{2}, R_{p}\right)$ for Arbre and Hilbert, respectively, $T(x)$ is the transfer function (15) or (21) calculated using $x$, ydata is the collected frequency response to fit to (15) or (21), $T(x)_{i}$ and $y d a t a_{i}$ are the simulated and collected responses at frequency $\omega_{i}$, and $n$ is the total number of data points in the collected frequency response. This routine aims to find the impedance parameters that would ideally reduce the least squares error to zero. The non-linear least squares fitting parameters extracted from the experimental results are given in Table VIII. The MATLAB simulated responses using these extracted parameters compared to the experimental results are shown in Fig. 17.

Both sets of MATLAB simulated frequency responses show very good agreement with the experimental results. We note that the extracted order of the Arbre FC is very close to the expected 0.5 [26] but that there is a deviation of 0.2589 from the expected order of the Hilbert FC. This deviation could possibly be attributed to damage incurred to the device in its shipping. Regardless, the use of these devices marks the first, to the authors best knowledge, physical realization of fractional filters without the use of an integer order approximation of a fractional capacitor or requiring an RC ladder realization. The successful implementation of fractional filters using manufactured two-terminal fractional capacitors in the Tow-Thomas biquad illustrates that the availability of these components commercially is approaching.

\section{Conclusion}

We have proposed modifying the traditional Tow-Thomas biquad to use fractional capacitors resulting in the realization of both fractional lowpass and asymmetric bandpass filters of order $0<\alpha_{1}+\alpha_{2} \leq 1$. The proposed asymmetric bandpass filters provide independent control of each stopband, not possible in previous filters, through manipulation of the fractional capacitor's orders. MATLAB and PSPICE simulations verify the fractional steps through the stopbands of the lowpass and bandpass fractional filters. We have also physically realized the fractional lowpass filter by using fractional capacitors implemented on silicon without requiring an approximation of $s^{\alpha}$. This work continues to build on 
the import of concepts from fractional calculus into analog circuit design highlighting their application in analog signal processing. We note that higher order filters with a fractional step are possible by cascading several Tow-Thomas biquad sections to obtain higher order filters [20].

\section{Acknowledgments}

The authors would like to acknowledge and thank Prof. Théodore Cissé Haba from the Départment Génie Electrique et Electronique at the Institut National Polytechnique in Yamoussoukro, Côte d'Ivoire for making available on loan the Arbre and Hilbert fractional capacitors. The first author would also like to acknowledge both Canada's National Sciences and Engineering Research Council (NSERC), Alberta Innovates - Technology Futures, and Alberta Advanced Education \& Technology for their financial support of this research through their graduate student scholarships.

\section{References}

[1] M. Ortigueira, "An introduction to the fractional continuous-time linear systems: the 21st century systems," IEEE Circuits Syst. Mag., vol. 8, no. 3, pp. 19-26, 2008.

[2] S. Das, Functional fractional calculus for system identification and controls, Springer, 2008.

[3] S. Westerlund, "Dead matter has memory! [capacitor model]," Phys. Scr., vol. 43, no. 2, pp. 174-179, 1991.

[4] S. Westerlund and L. Ekstam, "Capacitor theory," IEEE Transactions on Dielectrics and Electrical Insulation, vol. 1, no. 5, pp. 826-839, 1994.

[5] I. Podlubny, I. Petras, B. Vinagre, P. O'Leary, and L. Dorcak, "Analogue realizations of fractional-order controllers," Nonlinear Dynamics, vol. 29, pp. 281-296, 2002.

[6] T. Haba, G. Loum, J. Zoueu, and G. Ablart, "Use of a component with fractional impedance in the realization of an analogical regulator of order 1/2," J. Applied Sciences, vol. 8, no. 1, pp. 59-67, 2008.

[7] J. Machado, I. Jesus, A. Galhano, and J. Cunha, "Fractional order electromagnetics," Signal Processing, vol. 86, no. 10, pp. 2637-2644, 2006.

[8] F. Miguel, M. Lima, J. Machado, and M. Crisostomo, "Experimental signal analysis of robot impacts in a fractional calculus perspective," J. of Advanced Computational Intelligence and Intelligent Informatics, vol. 11, p. 1079, 2007.

[9] N.F. Ferreira, F. Duarte, M. Lima, M. Marcos, and J.T. Machado, "Application of fractional calculus in the dynamical analysis and control of mechanical manipulators," Fractional Calculus and Applied Analysis, vol. 11, no. 1, pp. 91-113, 2008.

[10] A. Elwakil, "Fractional-order circuits and systems: An emerging interdisciplinary research area," IEEE Circuits Syst. Mag., vol. 10, no. 4, pp. 40-50, 2010.

[11] A. Radwan, A. Soliman, and A. Elwakil, "First-order filters generalized to the fractional domain," Journal of Circuits, Systems and Computers, vol. 17, no. 1, pp. 55-66, 2008.

[12] A. Radwan, A. Elwakil, and A. Soliman, "On the generalization of second-order filters to the fractional-order domain," Journal of Circuits, Systems and Computers, vol. 18, no. 2, pp. 361386, 2009.

[13] A. Lahiri and T.K. Rawat, "Noise analysis of single stage fractional-order low pass filter using stochastic and fractional calculus," ECTI Transactions on Electrical Eng., Electronics, and Communications, vol. 7, no. 2, pp. 136-143, 2009.

[14] T. Freeborn, B. Maundy, and A. Elwakil, "Second order approximation of the fractional laplacian operator for equal-ripple response," in 2010 IEEE Midwest Symposium on Circuits and Systems, pp. 1173-1176, 2010.

[15] T. Freeborn, B. Maundy, and A. Elwakil, "Field programmable analogue array implementation of fractional step filters," IET Circuits, Devices and Systems, vol. 4, no. 6, pp. 484-491, 2010.

[16] P. Ahmadi, B. Maundy, A. Elwakil, and L. Belostotski, "Band-pass filters with high quality factors and asymmetric-slope characteristics," in 2011 IEEE Midwest Symposium on Circuits and Systems, pp. 1-4, 2011. 
[17] J. Tow, "Active rc filters-state-space realization," IEEE Proceedings, vol. 56, no. 6, pp. 1137$1139,1968$.

[18] L. Thomas, "The biquad: Part i - some practical design considerations," IEEE Trans. Circuit Theory, vol. CT-18, no. 3, pp. 350-357, 1971.

[19] L. Thomas, "The biquad: Part ii - a multipurpose active filtering system," IEEE Trans. Circuit Theory, vol. CT-18, no. 3, pp. 358-361, 1971.

[20] B. Maundy, A. Elwakil, and T. Freeborn, "On the practical realization of higher-order filters with fractional stepping," Signal Processing, vol. 91, no. 3, pp. 484-491, 2011.

[21] J.-S. Hong and M. Lancaster, "Microstrip cross-coupled trisection bandpass filters with asymmetric frequency characteristics," IEE Proc., Microw. Antennas Propag., vol. 146, no. 1, pp. 8490, 1999.

[22] P. Field, I. Hunter, and J. Gardiner, "Asymmetric bandpass filter using a novel microstrip circuit," IEEE Microw. Guid. Wave Lett., vol. 2, no. 6, pp. 247-249, 1992.

[23] A. Radwan, A. Soliman, A. Elwakil, and A. Sedeek, "On the stability of linear systems with fractional-order elements," Chaos, Solitons and Fractals, vol. 40, no. 5, pp. 2317-2328, 2009.

[24] B. Krishna and K. Reddy, "Active and passive realization of fractance device of order 1/2," Active and Passive Electronic Components, 2008.

[25] T. Haba, G. Ablart, and T. Camps, "The frequency response of a fractal photolithographic structure," IEEE Trans. Dielectr. Electr. Insul., vol. 4, no. 3, pp. 321-326, 1997.

[26] T. Haba, G. Ablart, T. Camps, and F. Olivie, "Influence of the electrical parameters on the input impedance of a fractal structure realised on silicon," Chaos Solitons Fractals, vol. 24, no. 2, pp. 479-490, 2005.

[27] G. Ablart, T. Haba, and G. Loum, "An analytical expression for the input impedance of a fractal tree obtained by a microelectronical process and experimental measurements of its non-integral dimension," Chaos Solitons Fractals, vol. 33, no. 2, pp. 364-373, 2007.

[28] I.S. Jesus, J.A.T. Machado, and M.F. Silva, "Fractional order capacitors," Proceedings of the IASTED International Conference on Modelling, Identification, and Control, MIC, pp. 346-351, 2008.

[29] B.B. Mandelbrot, The Fractal Geometry of Nature, New York: W.H. Freeman and Company, 1983.

[30] J. Clerc, A.-M. Tremblay, G. Albinet, and C. Mitescu, "A.c. response of fractal networks," Journal de physique. Lettres, vol. 45, no. 19, pp. 913-924, 1984. 